\title{
Game Theory for Resource Allocation in Heterogeneous Wireless Networks - A Review
}

\author{
Farhat Anwar, Mosharrof H. Masud, Burhan ul Islam Khan, Rashidah F. Olanrewaju, \\ Suhaimi A. Latif \\ Department of Electrical and Computer Engineering, Kulliyyah of Engineering, International Islamic University \\ Malaysia, Kuala Lumpur, Malaysia
}

\begin{tabular}{l} 
Article Info \\
\hline Article history: \\
Received May 21, 2018 \\
Revised Jul 22, 2018 \\
Accepted Aug 5, 2018 \\
\hline
\end{tabular}

Keywords:

Game theory

Heterogeneous wireless

networks

Resource allocation

Shapley value

\section{Corresponding Author:}

Burhan ul Islam Khan,

Department of Electrical and Computer Engineering, Kulliyyah of Engineering,

International Islamic University Malaysia,

Kuala Lumpur, Malaysia.

Email: burhan.iium@gmail.com

\begin{abstract}
In next-generation wireless networks, a user can be connected through MultiMode Device (MMD) to the multiple wireless networks in Heterogeneous Wireless Networks (HWN) considering several factors; including network technology, data service type, available bandwidth, Quality of Service (QoS), monetary cost, etc. To deal with all these multi attributes, game theory based models have been used to point out a better solution. This paper evaluates the techniques, methods, advantages, limitations of some game theory-based models for wireless resource allocation in HWN. Finally, it concludes that the Shapley Value method can be used for further research activities for its efficiency.
\end{abstract}

Copyright $\odot 2018$ Institute of Advanced Engineering and Science. All rights reserved.

\section{INTRODUCTION}

The next generation wireless networks are envisaged to be connected through multiple wireless links like 3G, WiFi, WiMAX and LTE, etc. at anytime and anywhere with the best quality of service that creates heterogeneous wireless environments. These varieties of wireless links give the options to the users of being "always best connected" where the system attempts keeping the users linked to the Internet all the time [1]. The aforementioned wireless technologies were developed to be operated individually. Due to the advancement of the wireless communications, multi-homing supportive Mobile Devices (MD) have been introduced [2]. The primary resource of the wireless link is bandwidth that has been allocated from a wireless link to the MD. However, the bandwidth of a single link may not be always enough to operate the heavy traffic, particularly for real-time traffic like video conferencing. Hence, the available resources or bandwidth in a particular area from all available links can be integrated, and the traffic can be transmitted [3]. The most critical issue includes determining the most appropriate network for a particular user in its present situation [1]. The network selection criteria depend on many factors, such as security, available throughput, cost, delay, jitter, reliability etc. [4], [5].

In HWN environment [6], the MD has the options to select the best suitable AP for its needs based on its preferences. Considering the multiple scenarios in HWN including the type of users, technology, service provider and applications, require the development of the new dimension that offers dynamic automatic networks selection [7]. Many solutions have been proposed to address this multi-criteria decisionmaking algorithms in HWN. Game theory [8] also can be used to deal with the complex decision making between the networks and the mobile users for resource allocations in HWN [9] environment. 


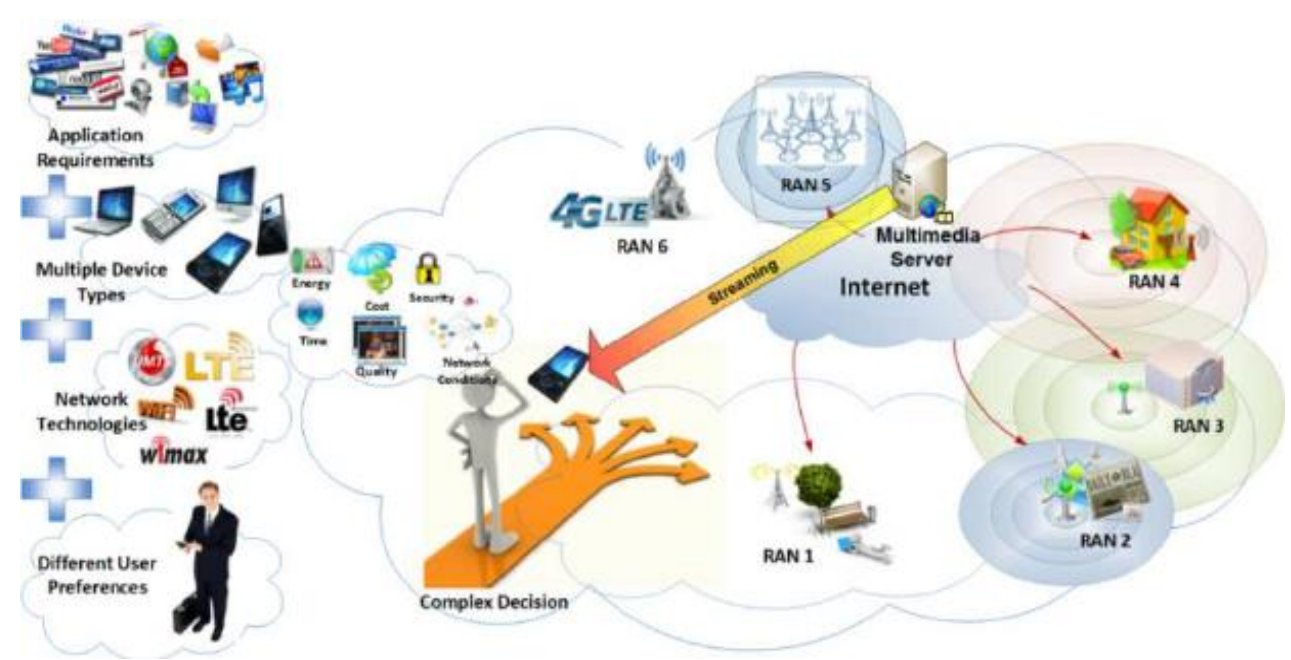

Figure 1. Heterogeneous Wireless Networks Environment [6]

\section{HETEROGENEOUS WIRELESS NETWORK}

Heterogeneous Wireless Network (HWN) may be defined as the combination of two or more wireless resources like Wireless Fidelity (WiFi), World Interoperability for Microwave Access (WiMAX) and Global System for Mobile communication (GSM) in a typical area as shown in Figure 1. A mobile device enabled with multiple interfaces can have access to any such resource on the basis of its application demand that runs on the Mobile Node (MN). The most common and accessible wireless technology comprises of the cellular technology followed by the WiFi and WiMAX technologies. Cellular technology has a large coverage span but smaller bandwidth, WiFi has lesser coverage, but high bandwidth and WiMAX is recognised for high bandwidth as well as area of coverage.

Currently, various network technologies like WiFi or IEEE $802.11 \mathrm{a} / \mathrm{b} / \mathrm{g}$, WiMAX or IEEE 802.16, UMTS, GPRS are merging their infrastructures with the core networks of IPv6 or IPv4. All the access technologies involved with HWN possess their individual features such as QoS support, operational costs and coverage [10]. The mobile nodes enabled with multiple interfaces may be linked to a proper interface on the basis of the requirements of the application on the mobile node and network strength [11]. The primary goal of the HWN is the capability of a mobile node to retain its present session and choose the most suitable interface while it is communicating. Every technology has its particular set of policies and rules that govern the provision of services and resources to its users. Thus, a significant issue in HWN is the design of a Radio Resource Management (RRM) system that is efficient. In general, the RRM framework may be apportioned using the functionalities viz. Decision Enforcement, Decision Making and Resource Monitoring. These functionalities are interrelated in such a way that the results of resource monitoring are employed in decision making after which decision enforcement takes place. Different solutions have been adopted to make this type of complicated decision and allocate the wireless resources into the MN where game theory has been given significant attention in recent years.

\section{GAME THEORY MODEL}

Game theory is a mathematical tool employed in designing and modeling of complex scenarios that involve the interaction of reasonable decision makers with feasibly conflicting mutual interests [7]. It was initially invented to solve the complex issue of economic behaviour. It is popular in other fields including politics, philosophy, military, sociology and telecommunication due to the effectiveness of its studying complex dynamics among players [12]. Recently, a large number of issues in wireless communications and networking, particularly security [13], [14], [15] have been addressed using game theory and its solution. Wireless resources are insufficient in terms of capacity, power and bandwidth. On the other hand, resource scarcity and the rising figure of wireless access terminals and result in severe contest among mobile customers for required wireless resources. In this context, game theory can provide significantly better understanding and optimum allocation of resources among the players. In recent times, game theory has been investigated to address wireless communication issues including routing, resource allocation, power control MIMO systems [16], load control, medium access control, etc. [7]. A classification has been performed based on OSI layer (Transport, Network, Data link, Physical) in light of game theoretic 
approaches [17]. A detailed discussion has been covered in a recent book on a vast area of networking domains and wireless communications including sensor networking, vehicular networking, power control system and radio resource management [18]. Heterogeneous wireless communication networks are dynamic in terms of network load, availability, energy conservation [19], [20], monetary cost and network coverage [21]. Both operators and users seek to maximise their payoffs. The payoffs may be projected using utility functions on the basis of several decision criteria from both sides where game theory can be well suited.

\subsection{Basic Concept of Game Theory}

Game theory comprises three chief components, the player set, the action set and the payoff set. It is a common interest of the players to raise their payoffs to the maximum by adopting appropriate strategic actions available in a specific moment. The combination of the best-chosen strategy for every player is referred to as equilibrium. In a circumstance when a player is not able to gain by modifying his strategic actions while keeping the strategies of other players similar, then the game is named as Nash Equilibrium. The payoff for every player may be distributed based on the estimated utility they have played for that specific set of strategies. On the other hand, if the payoffs may not be improved further with another combination of strategies, it can be said that the game has attained a Pareto Optimal Nash Equilibrium [7].

a) Nash Equilibrium: Suppose $N$ is the number of players in a game, and i is an index of a player where $0<i \leq N$. Suppose $S_{i}$ reperesents the set of mixed strategies available for player i with $s_{i} \in S_{i}$ being a feasible strategy for player i. The Nash Equilibrium meets the condition shown in Equation (1).

$$
\pi_{i}\left(s_{i}^{*}, s_{-}^{*}\right) \geq \pi_{i}\left(s_{i}^{*}, s_{-}^{*} i\right) \forall 0<i \leq N \forall s_{i} \in S_{i}
$$

Where, $\pi_{i}()$ is the payoff function of player $\mathrm{i}, s_{i}^{*}$ denotes a Nash Equilibrium strategy of player $\mathrm{i}$, and $s_{i-1}^{*}$ represents the Nash Equilibrium strategies of every player in the set excluding player $i$. Nevertheless, several games possibly will not have a Nash Equilibrium, otherwise they may not have just one Nash Equilibrium.

b) Pareto Optimal Nash Equilibrium: Suppose $\mathrm{N}$ is the total number of game players, and $\mathrm{i}$ is the player index with $0<i \leq N$. Suppose $S_{i}$ represents the set of mixed strategies available for player i with $S_{i}^{*}$ denoting the Nash Equilibrium strategy set for player i, $s_{i}^{*} \in S_{i}^{*}$. The Pareto Optimality meets the condition shown in Equation (2).

$$
\pi_{i}\left(s_{i}^{p}\right)>\pi_{i}\left(s_{i}^{p}\right) \forall 0<i \leq N \forall s_{i}^{*} \in S_{i}^{*}
$$

Where $\pi_{i}()$ denotes the payoff function associated with player i and $S_{i}^{p}$ represents the strictly Pareto Optimal strategy.

The correlation between game theory and wireless networks has been tabulated in Table 1 .

\begin{tabular}{|c|c|c|}
\hline $\begin{array}{c}\text { Game } \\
\text { Components }\end{array}$ & Concept in Real Game & Concept in Wireless Networks \\
\hline Players & The players involved in the game & The agents involved in the game like networks and user. \\
\hline Strategies & $\begin{array}{l}\text { A set of actions to be performed by the } \\
\text { players while playing the game }\end{array}$ & $\begin{array}{l}\text { A set of actions to be performed by the players while playing the } \\
\text { game: available networks, network strength, available bandwidth, } \\
\text { offered prices, subscription plans etc. }\end{array}$ \\
\hline Payoffs & The rewards received by the players & $\begin{array}{l}\text { The profit of the players estimated based on the utility functions: } \\
\text { QoS, Network capacity, etc. }\end{array}$ \\
\hline Resources & Fame, Money & For which the players are competing like bandwidth, power, etc. \\
\hline
\end{tabular}

Table 1. Correlation between Game Theory and Wireless Networks

\subsection{Benefits of Game Theory in HWN}

Game theory provides particular advantages for analyzing protocols and distributed algorithms in wireless networks where few advantages have been addressed in this section [22].

a) Distributed system analysis: When network nodes carry out independent adaptations, game theory permits the investigation of convergence, inimitability and existence to a balanced-state operation point. Therefore, it is a powerful tool for precise investigation of distributed protocols.

b) Cross-layer optimisation: Usually in ad-hoc online games, the node decision at a specific level is to optimise the performance of some other layer. By devising the action space properly, game theory analysis may provide an understanding about the cross-layer optimisation methods. 
c) Incentive Plan Design: Mechanism design forms a part of game theory. It focuses on how to design incentive mechanisms to guide independent, self-interested participants in obtaining the desired results from a system-wide perspective. It could be particularly useful in the designing of incentive plans for ad hoc networks.

\subsection{Challenges of Game Theory in HWN}

In heterogeneous wireless network environments, users are expected to possess multiple-mode wireless mobile devices and can choose to link to one or many access networks with different technologies, based on the preferences of the coverage area, available bandwidth, service providers, and monetary costs [22]. Considering these issues in mind, the game theory approach has been used to simulate and evaluate the competitive interaction or cooperation between logical decision makers representing network operators and/or users.

One of the significant tasks is to determine the appropriate game strategy, whether the player is cooperative or non-cooperative, and define the problem accordingly. Strategies must be identified and adopted among players during critical game time. It has been known that game theory works as a hypothesis of reasonableness and players to act in their best interests. Besides, service providers try to raise their revenue by multiplying the number of users, and users anticipate the best quality of service (QoS) from the provider. However, the supplier's profit maximisation or client's best QoS cannot be guaranteed.

By using game theory models, rational models can be designed to allow players to compete with each other and seek to maximise their profits. By using collaborative game models to analyse some scenarios, they find themselves selfish and refuse to cooperate to maximise their profits. Considering this situation, to improve the overall QoS of the network, an incentive mechanism has been proposed and adopted. The purpose of using incentives is to inspire players to work together to maximise social welfare. However, due to the dynamic nature of the wireless environment, random wireless errors and overall degraded interference or packet collisions that result in QoS in the network, cooperative methods may not be suitable.

Another important aspect is how participants decide: a distributed or centralised approach. Centralized methods are rarely used in HWN since the computational cost increases as the network scale increases. On the whole, game theory is more applicable to self-configuring facility allocation methods.

\section{GAME THEORETIC MODEL FOR NETWORK SELECTION}

The players involved in the wireless game are wireless networks and mobile users. Both users and networks seek to raise their payoffs to the maximum by adopting available strategies for a specific moment. For example, users mainly seek good quality with affordable cost while the networks look for the maximisation of monetary profit. Benefits can be assessed by means of utility functions using various decision criteria: energy savings, monetary costs, availability, network load, and so on [7, 23]. The game theory has been investigated in recent years and can be classified in mainly two ways, cooperative and noncooperative model.

The approaches can be varied from many issues including the game models (evolution games, auction games, bargain games, repeated games, etc.), players (networks or users), strategies (service requests, available APs, transmission rates, etc.), game pools (single or multiple operations), pricing schemes (dynamic or flat rate pricing), set of parameters such as latency, monetary cost, jitter, packet loss, throughput, etc., use of RATs (Cellular, WiMAX, WLAN). Nevertheless, the chief goal of the game is the same: network selection is a problem of resource allocation [7].

\subsection{Cooperative Game Model}

When a group of players forms a "consortium" and completes between player leagues, rather than between individual players, cooperative game theory can be defined [24]. Cooperative games include a list of players and characteristic function. Let, $\mathrm{N}$ represents a player set who form a coalition and $\mathrm{v}$ is the characteristic functions. Formally, cooperative game is a pair of $(\mathrm{N}, \mathrm{v})$, with $\mathrm{N}=\{1, \ldots \ldots \mathrm{n}\}$ is a finite player set, and $\mathrm{v}$ denotes characteristic function $\mathrm{v}$ : $2 \mathrm{n} . .>\mathrm{R}$, e.g., $\mathrm{v}(0)=0$. For an $\mathrm{n}$ player game, the number of coalitions possible are $2 \neg n$.

Different types of cooperative game theoretic models have been proposed in recent years. Some of the popularly utilized game theoretic models have been reviewed in this paper.

a) Strategic Game: A unique strategic planning that has been taken only once by a player where other players are unaware of this player's action. The players are independent, and they choose their action simultaneously. The famous strategic game is Prisoner's Dilemma [25]. 
The prisoner's dilemma presents a situation where there are two suspects for accomplishing a huge crime and detained in two different cells. Table 2 shows the benefits of this game; the concept is that players are trying to reduce their imprisonment to the minimum. If they are all quiet (cooperative with each other), everyone shall be sentenced to an imprisonment of one year. If they all admit (defects), everyone will be sentenced to three years imprisonment.

\begin{tabular}{ccc}
\multicolumn{3}{c}{ Table 2. Prisoner's Dilemma Payoff Table } \\
\hline & Defect & Cooperate \\
\hline Defect & 3,3 & 0,4 \\
Cooperate & 4,0 & 1,1 \\
\hline
\end{tabular}

If only one of them confesses, he/she will be released, and another witness shall be sentenced to four years imprisonment. The best result for players is that they all cooperate, which means they don't admit it, but each of them has an incentive to try to get rid of the jail's "free ride" (defects). In seclusion, both parties will choose to cooperate with defects, resulting in unique Nash equilibrium (defects, defects) in the game.

The chief concept of Repeated Game is to identify the strategic pattern of previous games and associations among the players. The players make decisions based on the previous experience of strategic plans that have been adopted by other players. If we assume the repeated prisoner's dilemma game, having a similar payout table as shown in Table 2. Although the cooperation of the two players is better, for each player, the players' flaws strictly control the player's cooperation. Therefore, when each player is defective, the game has an inimitable Nash equilibrium. When the game is repeatedly played, the desired result of each other is obtained once they are cooperative in each period (long-term return). If every player thinks they will lead to the end of cooperation, this will lead to short-term gains, but it will lead to long-term losses, which will become stable.

b) Bankruptcy Game: The game theoretic model is used to solve the distribution problems to allocate the resources among the group of agents. It is well suited in a circumstance where the resources (bandwidth) are insufficient to satisfy all agents (mobile users). To understand the concept of game theory, Suppose a company owning E property goes bankrupt and owes an amount $\mathrm{d}$ to creditors. Therefore, money $\mathrm{E}$ needs to be distributed among $\mathrm{N}$ creditors. In the worst case, the creditor's total debt is greater than the debt of the insolvent company, $\mathrm{E}<=\mathrm{d}$. This contradictory situation presents an $\mathrm{N}$-person cooperation game [26], in which game agents seek equilibrium to allocate money [27]. The allocation can be done using the following set of rules. The bankruptcy game uses three different partitioning rules to solve: Constraint Equal Awards (CEA) rules - rewards are as equal as possible, follow the principle of first come first serve; Random Arrival (RA) rules and Talmud rules - if the total (bandwidth) is less than half the summation of the claims, then the CEA rules are employed and operated on the semi-claims, otherwise, if it is greater, then the Constrained Equal Loss (CEL) rules are used to balance the losses. The results indicate that the total capacity maximization is achieved by employing CEA but the Talmud and RA rules behave better when it comes to maximum fairness [7].

\subsection{Non-cooperative Game Model}

In the non-cooperative game model, users compete with the network, and each network seeks to raise their own utility to the maximum. Users try to get the maximum service income from the price they pay. Then again, the network attempts to maximise the profits of the services it provides.

The non-cooperative game theory based network model is designed as a non-cooperative auction game, that consists of three parts: bidder, seller and auctioneer. The user represents the buyer, the seller/bidder is similar to the network operator available, and the auction item is signified by the bandwidth requested with the relevant features. Calculate the winning bid, for example; it will maximise the utility of the user. The collaboration between the networks and the users has been investigated. The author classifies the problem of network selection as a non-cooperative auction game. The game has three parts: the bidder, the seller, and the auctioneer [28], [29], [30].

In another study, the authors presented a non-cooperative game theory based wireless resource management framework. The framework consists of four essential constituents: connection level allocation, network-level allocation, admission control, and capacity reservation [31]. The problem of bandwidth allocation was simulated as non-cooperative game among various access networks. The solution to this problem was acquired from Nash equilibrium, indicating that the overall utility of the network is maximised. The bargaining game is utilised to simulate the problem of capacity reservation. Connection-level assignments were exhibited as trading market games, and the solution to the game was observed to be Nash equilibrium [32]. 
It has been suggested that non-cooperative multi-phase games between two self-governing wireless LANs should simulate access control problems [33]. The game players comprise of two networks, and the user's service request comprises of their policy set. The outcome of the game is the dispersal of service requests all over the network so that every network gets the most return [34], the summarizes the cooperative and non-cooperative shown in Table 3

Table 3. Summary of Cooperative and Non-cooperative Game Models

\begin{tabular}{|c|c|c|c|c|}
\hline Game Type & Game Model & Objectives & Parameters & Limitations \\
\hline \multirow{3}{*}{ Cooperative } & $\begin{array}{l}\text { Strategic Game } \\
\text { Model }\end{array}$ & $\begin{array}{l}\text { To maximise each } \\
\text { player's payoffs. }\end{array}$ & $\begin{array}{l}\text { The players are independent } \\
\text { and the plan can only be } \\
\text { performed once. }\end{array}$ & $\begin{array}{l}\text { If one player benefits, } \\
\text { others will become } \\
\text { losers. }\end{array}$ \\
\hline & $\begin{array}{l}\text { Repeated Game } \\
\text { Model }\end{array}$ & $\begin{array}{l}\text { To identify the } \\
\text { pattern of previous } \\
\text { games. }\end{array}$ & $\begin{array}{l}\text { Repeat the game based on } \\
\text { experience. }\end{array}$ & $\begin{array}{l}\text { Short-term gains, but } \\
\text { long-term losses. }\end{array}$ \\
\hline & $\begin{array}{l}\text { Bankruptcy } \\
\text { Game Model } \\
\text { (Shapley Value) }\end{array}$ & $\begin{array}{l}\text { To allocate the } \\
\text { resources among the } \\
\text { groups of agents. }\end{array}$ & $\begin{array}{l}\text { The creditor's total debt is } \\
\text { greater than the bankrupt } \\
\text { company's money. }\end{array}$ & $\begin{array}{l}\text { Ideal for multi-attribute } \\
\text { components. }\end{array}$ \\
\hline \multirow{3}{*}{$\begin{array}{l}\text { Non- } \\
\text { cooperative }\end{array}$} & $\begin{array}{l}\text { Interaction } \\
\text { between } \\
\text { networks }\end{array}$ & $\begin{array}{l}\text { Total network utility } \\
\text { is maximised }\end{array}$ & Bargaining Game & $\begin{array}{l}\text { Not all components justly } \\
\text { evaluated. }\end{array}$ \\
\hline & $\begin{array}{l}\text { Multi-stage } \\
\text { game }\end{array}$ & $\begin{array}{l}\text { Gains the maximum } \\
\text { payoff for overall } \\
\text { networks. }\end{array}$ & Users' service requests & $\begin{array}{l}\text { Without proper } \\
\text { consideration of proper } \\
\text { importance, it may } \\
\text { produce the worst results. }\end{array}$ \\
\hline & $\begin{array}{l}\text { Multi-strategy } \\
\text { game }\end{array}$ & $\begin{array}{l}\text { To maximise its } \\
\text { own payoff } \\
\text { individually. }\end{array}$ & Individual preferences & $\begin{array}{l}\text { These ideas are not } \\
\text { assessed, nor do they } \\
\text { show progress. }\end{array}$ \\
\hline
\end{tabular}

Network selection is modelled by another study as a non-cooperative game with multiple stages [35]. Players have three wireless access networks: WiMAX, WLAN, and WCDMA. This policy set is signified by the user's service request, and calculates the strength of the revenue network for every network on the basis of the type of service (surfing the Internet, voice call or streaming video), user preferences (quality and cost), traffic status and signals (bad, medium or good), user's speed (low, stable or high) and battery drainage rate. The result of the game is the dissemination of service requests across the network, and every network attempts to maximise its revenue [35].

\section{GAME THEORY MODEL IN HWN}

A new mechanism has been proposed in this thesis for a fresh connection that may be served by available networks in that particular area simultaneously. Let us assume three different network technologies are available, namely WLAN (WiFi), Cellular network and WMAN (WiMAX). A Multimode Mobile Device (MMD) initiates the new request with the required bandwidth (estate) to be allocated from different networks. This scenario is quite similar to the bankruptcy game theory where each of the networks tries to maximise its utilities by providing sufficient bandwidth required for gaining revenue from the fresh connection [31]. Here, $\mathrm{N}(=3)$ is the total number of agents and the agent set is defined as $S=\{$ wifi, cel, wimax \}.

When a request for bandwidth $\mathrm{B}$ is sent by a new connection, a central radio network controller decides the expanse of bandwidth offered to that connection from every network. The bandwidth offered changes with time and application to application based on the subscription policies. Notably, the bandwidth offered may be defined by Equation (3) as follows [25]:

$$
d_{i}=\left\{\begin{array}{l}
b_{i}^{k} \\
\left(B_{i}^{a v}\right)^{r}+N\left(B_{i}^{a v}-\left(B_{i}^{a v}\right)^{r}\right)
\end{array}\right\} \begin{aligned}
& b_{i}^{k}<\left(B_{i}^{a v}\right)^{r} \\
& b_{i}^{k, r e q} \geq\left(B_{i}^{a v}\right)^{r}
\end{aligned}
$$

Where, $b_{i}^{k}$ represents the predefined bandwidth to a fresh connection from network $\mathrm{i}$ for $\mathrm{k}$ type of services. $\left(B_{i}^{a v}\right)$ denotes the bandwidth available in the network i. $b_{i}^{k, r e q}$ signifies the expanse of bandwidth requested by a fresh connection of $\mathrm{k}$ types of class. $\mathrm{N}$ represents the uniform random number ranging from 0 to 1 and $\mathrm{r}$ specifies the control parameter that shall be called the bandwidth shaping parameter. It can be noted that bandwidth $b_{i}^{k}$ can be allocated from i network to a fresh connection in regular traffic load when 
$b_{i}^{k}<\left(B_{i}^{a v}\right)^{r}$ satisfies. However, in a congested traffic scenario, when bandwidth requested is greater than the bandwidth available $b_{i}^{k, r e q} \geq\left(B_{i}^{a v}\right)^{r}, \mathrm{~N}$ and the shaping parameter for ensuring that the network does not provide excessive bandwidth to the fresh connection.

When a single network cannot serve a user, a model has been proposed that enables the user to divide the application traffic among the coalition members using a cooperative Stackelberg. It is a characteristic function that is employed for expressing the coalition payoff, and the main concept is utilized for analyzing the allocation stability.

Shapley Value [36] is one of the fairest models to solve the $N_{\mathrm{i}}^{\mathrm{k}}$ person bankruptcy game due to the less computational complexity [32]. It proposes the most equitable distribution of the profits attained collectively among the collaborative game players. The primary focus is to realize the relative contribution of each player.

For computing Shapley Value, suppose a function $\varnothing(v)$ is defined that represents the value or worth of player $i$ in the game with a characteristic function. If a player takes part in the coalition at random, the average payoff to a player is the Shapley Value. The characteristic function $v(S)$ can be calculated as follows [31] as mentioned in Equation (4).

$$
v(S)=\max \left(0, B_{i}^{k}-\sum_{j \not \subset S} d_{j}^{k, \max }\right)
$$

For all possible coalitions of $\mathrm{S}$.

Where $B_{i}^{k}$ indicates the total bandwidth required to transmit $\mathrm{k}$ types of services from $\mathrm{i}$ types of networks. $\sum_{j \not S} d_{j}^{k, \max }$ is the sum of the offered bandwidth from user $\mathrm{j}$ as depicted in Equation (5).

$$
\phi_{i}(v)=\sum \frac{(|s|-1) !\left(N_{i}^{k}-|s|\right) !}{N_{i}^{k} !}[v(S)-v(S-\{i\})]
$$

Where $|s|$ signifies the total players in the coalition assuming the $j$ th user is in the coalition, $N_{i}^{k}$ represents the overall number of players, $v(S)$ specifies the coalition utility taking in player $i$, and $v(S-\{i\})$ refers to coalition utility without player i. The Shapley value, $\emptyset_{i}(v)$ can be obtained from Equation (5) by changing the value of $i=1,2,3, \ldots N_{i}^{k}$ and thus, the value of bandwidth allocation $d_{j}^{k}$ may be calculated.

The other notations and descriptions of the variables involved have been given in

\begin{tabular}{|c|c|c|}
\hline Variable & Bankruptcy Game & Bandwidth Allocation \\
\hline$N_{i}^{k}$ & Overall number of creditors & Total number of networks \\
\hline$B_{i}^{k}$ & The estate (money) of the company & $\begin{array}{l}\text { Requested bandwidth for kth category of services from ith } \\
\text { network }\end{array}$ \\
\hline$s$ & Set of agents & Set of networks \\
\hline$d_{j}^{k, \max }$ & The maximum claim of the jth creditor & The jth user's maximum bandwidth demand \\
\hline$d_{j}^{k, \min }$ & The minimum claim of the jth creditor & The jth user's minimum bandwidth demand \\
\hline$d_{j}^{k}$ & The actual money disbursed for the jth user & Bandwidth allocation to the new connection in the network $\mathrm{i}$. \\
\hline
\end{tabular}

Table 4. Notations and Descriptions of Variables used

\section{DISCUSSION AND CONCLUSION}

In HWN environment, users aim to be equipped with Multi-Mode Device (MMD) to be connected with one or many wireless access networks in a particular coverage area based on several factors including; type of technology, available bandwidth, monetary cost, etc. Considering these multi-attribute decisionmaking issues, game theory approaches have been utilised for modelling and analysing the competitive or cooperative interaction among rational decision makers. The most challenging issues for the players is to determine the problem, whether the game is cooperative or a non-cooperative. In HWN, there are many combinations of competition among players like networks vs networks, users vs users and networks vs users. The users seek the best quality of service from the networks that they have paid for and the networks try to maximise its revenue by increasing the number of users. However, it is not always guaranteed that both parties will be benefited equally.

It can be noted from the review as mentioned earlier that, cooperative game players are supposed to be collaborating for maximising their group payoffs, and non-cooperative game players try to profit or

Game Theory for Resource Allocation in Heterogeneous Wireless Networks - A Review (Farhat Anwar) 
conserve their limited individual resources. Finally, it can be said that the Shapely Value method is generally employed in multi-attribute decision-making field to reach a concrete solution.

\section{ACKNOWLEDGEMENTS}

This work was partially supported by Ministry of Higher Education Malaysia (Kementerian Pendidikan Tinggi) under Fundamental Research Grant Scheme (FRGS) number FRGS13-081-0322.

\section{REFERENCES}

[1] Gustafsson E, Jonsson A. Always best connected. IEEE Wireless communications. 2003 Feb; 10(1): 49-55.

[2] Masud MH, Latif SA, Alam FA. A scheduling algorithm for bandwidth aggregation in Heterogeneous Wireless Network. 2014 International Conference on Informatics, Electronics \& Vision (ICIEV). IEEE. 2014 May 23: 1-4.

[3] Latif SA, Masud MH, Anwar F, Alam MK. An investigation of scheduling and packet reordering algorithms for bandwidth aggregation in heterogeneous wireless networks. Middle-East Journal of Scientific Research. 2013; 16(12): 1613-1623.

[4] Song Q, Jamalipour A. A network selection mechanism for next generation networks. IEEE International Conference on Communications, ICC 2005. IEEE. 2005 May 16; 2: 1418-1422.

[5] Fux V, Maillé P. A rating-based network selection game in heterogeneous systems. 8th EURO-NGI Conference on Next Generation Internet (NGI). IEEE. 2012 Jun 25: 125-132.

[6] Trestian R, Ormond O, Muntean GM. Performance evaluation of MADM-based methods for network selection in a multimedia wireless environment. Wireless Networks. 2015 Jul 1; 21(5): 1745-1763.

[7] Trestian R, Ormond O, Muntean GM. Game theory-based network selection: Solutions and challenges. IEEE Communications surveys \& tutorials. 2012 Oct; 14(4): 1212-1231.

[8] Morganstern O, Von Neumann J. The theory of games and economic behavior. Princeton University Press. 1944

[9] Maheswaran $\mathrm{CP}$, Sulochana $\mathrm{CH}$. Utilizing EEM approach to tackle bandwidth allocation with respect to heterogeneous wireless networks. ICT Express. 2016 Jun 1; 2(2): 80-86.

[10] Piamrat K, Ksentini A, Bonnin JM, Viho C. Radio resource management in emerging heterogeneous wireless networks. Computer Communications. 2011 Jun 15; 34(9): 1066-1076.

[11] Masud MH, Anwar F, Bari SM. A primary interface selection policy in heterogeneous networks based on QoS. $20124^{\text {th }}$ International Conference on Computer and Communication Engineering (ICCCE). IEEE. Kuala Lumpur, Malaysia. 2012 Jul 3: 706-711.

[12] Kim C, Langari R. Game theory based autonomous vehicles operation. International Journal of Vehicle Design. 2014; 65(4): 360-368.

[13] Khan BUI, Olanrewaju RF, Mattoo MU, Aziz AA, Lone SA. Modeling malicious multi-attacker node collusion in MANETs via game theory. Middle-East Journal of Scientific Research. 2017; 25(3): 568-579.

[14] Khan BUI, Olanrewaju RF, Anwar F, Shah A. Manifestation and mitigation of node misbehaviour in adhoc networks. Wulfenia Journal. 2014 Mar 3; 21(3): 462-470.

[15] Khan BUI, Olanrewaju RF, Mir RN, Baba A, Adebayo BW. Strategic profiling for behaviour visualization of malicious node in manets using game theory. Journal of Theoretical \& Applied Information Technology. $2015 \mathrm{Jul}$ 10; 77(1): 25-43.

[16] Olanrewaju RF, Khan BUI, Mir RN, Shah A. Behaviour visualization for malicious-attacker node collusion in MANET based on probabilistic approach. American Journal of Computer Science and Engineering. 2015; 2(2): 1017.

[17] Charilas DE, Panagopoulos AD. A survey on game theory applications in wireless networks. Computer Networks. 2010 Dec 20; 54(18): 3421-3430.

[18] Zhang Y, Guizani M, editors. Game theory for wireless communications and networking. $1^{\text {st }}$ ed. CRC press. 2011 Jun 23.

[19] Hassan S, Nisar MS, Jiang H. Energy preservation in heterogeneous wireless sensor networks through zone partitioning. Indonesian Journal of Electrical Engineering and Computer Science. 2016 May 1; 2(2): 390-395.

[20] Dhage MR, Vemuru S. A Effective Cross Layer Multi-Hop Routing Protocol for Heterogeneous Wireless Sensor Network. Indonesian Journal of Electrical Engineering and Computer Science. 2018 May 1; 10(2): 664-671.

[21] Zhang J, Wang Y, Liu Y, Li Q. Nodes Deployment Scheme of Heterogeneous Wireless Sensor Network Based on Organic Small Molecule Model. Indonesian Journal of Electrical Engineering and Computer Science. 2015 Dec 1; 16(3): 574-582.

[22] Srivastava V, Neel JO, MacKenzie AB, Menon R, DaSilva LA, Hicks JE, Reed JH, Gilles RP. Using game theory to analyze wireless ad hoc networks. IEEE Communications Surveys and Tutorials. 2005 Oct 1; 7(1-4): 46-56.

[23] Salih YK, See OH, Ibrahim RW. An intelligent selection method based on game theory in heterogeneous wireless networks. Transactions on emerging telecommunications technologies. 2016; 27(12): 1641-1652.

[24] Iturralde M, Wei A, Ali-Yahiya T, Beylot AL. Resource allocation for real time services in LTE networks: Resource allocation using cooperative game theory and virtual token mechanism. Wireless personal communications. 2013 Sep 1; 72(2): 1415-1435.

[25] Osborne MJ, Rubinstein A. A course in game theory. MIT press. 1994 Jul 12. 
[26] Pulido M, Sánchez-Soriano J, Llorca N. Game theory techniques for university management: an extended bankruptcy model. Annals of Operations Research. 2002 Jan 1; 109(1-4): 129-142.

[27] Chai R, Wang X, Chen Q, Svensson T. Utility-based bandwidth allocation algorithm for heterogeneous wireless networks. Science China Information Sciences. 2013 Feb 1; 56(2): 1-3.

[28] Khan MA, Toseef U, Marx S, Goerg C. Game-theory based user centric network selection with media independent handover services and flow management. Eighth Annual Communication Networks and Services Research Conference (CNSR). IEEE. 2010 May 11: 248-255.

[29] Khan MA, Toseef U, Marx S, Goerg C. Auction based interface selection with Media Independent Handover services and flow management. European Wireless Conference (EW). IEEE. 2010 Apr 12: 429-436.

[30] Khan MA, Sivrikaya F, Albayrak S, Mengal KQ. Auction based interface selection in heterogeneous wireless networks. 2nd IFIP Wireless Days (WD). IEEE. 2009 Dec 15: 1-6.

[31] Niyato D, Hossain E. A cooperative game framework for bandwidth allocation in $4 G$ heterogeneous wireless networks. IEEE International Conference on Communications, 2006. ICC'06. 2006 Jun; 9: 4357-4362.

[32] Charilas DE, Markaki OI, Vlacheas PT. Admission control as a non-cooperative multi-stage game between wireless networks. 16th International Conference on Systems, Signals and Image Processing, 2009. IWSSIP 2009. IEEE. 2009 Jun 18: 1-5.

[33] Charilas, D. E. and A. D. Panagopoulos, (2010) A survey on game theory applications in wireless networks. Elsevier Computer Networks. 54(18); 3421-3430.

[34] Niyato D, Hossain E. A noncooperative game-theoretic framework for radio resource management in 4G heterogeneous wireless access networks. IEEE Transactions on Mobile Computing. 2008 Mar; 7(3): 332-345.

[35] Ong EH, Khan JY, Mahata K. Radio resource management of composite wireless networks: Predictive and reactive approaches. IEEE Transactions on Mobile Computing. 2012 May; 11(5): 807-820.

[36] Shapley LS. A value for n-person games. In: Kuhn HW, Tucker AW, eds. Annals of Mathematics Studies 28. Princeton: Princeton University Press. 1953: 307-317. 